Pacific

Journal of

Mathematics

\title{
VERONESE SUBRINGS AND TIGHT CLOSURE
}

ANURAG K. Singh

Volume $192 \quad$ No. 2

February 2000 


\title{
VERONESE SUBRINGS AND TIGHT CLOSURE
}

\author{
ANURAg K. Singh
}

\begin{abstract}
We determine when an $\mathbb{N}$-graded ring has Veronese subrings which are F-rational or F-regular. The results obtained here give a better understanding of these properties, and include various techniques of constructing F-rational rings which are not F-regular.
\end{abstract}

\section{Introduction.}

Throughout this paper, all rings are commutative, Noetherian, and have an identity element. By a graded ring, we mean a $\operatorname{ring} R=\oplus_{n \geq 0} R_{n}$, which is finitely generated over a field $R_{0}=K$.

The theory of tight closure was developed by Melvin Hochster and Craig Huneke in [HH1], and has yielded many elegant and powerful results in commutative algebra and related fields. The theory draws attention to rings which have the property that all their ideals are tightly closed, called weakly $F$-regular rings, and rings with the weaker property that parameter ideals are tightly closed, called F-rational rings. The term F-regular is reserved for rings with the property that all their localizations are weakly F-regular. (The recent work of Lyubeznik and Smith shows that for graded rings the properties of weak F-regularity and F-regularity are equivalent, see [LS].) These properties turn out to be of significant importance, for instance the Hochster-Roberts theorem of invariant theory that direct summands of polynomial rings are Cohen-Macaulay ([HR1]), can actually be proved for the much larger class of F-regular rings.

While the property of F-rationality provides an algebraic analogue of the notion of rational singularities, F-regularity, in general, is not so well understood geometrically. One approach is to study the variety $X=\operatorname{Proj} R$ for a graded F-regular ring $R$. The Veronese subrings of $R$ are also homogeneous coordinate rings for $X$, and so it is interesting to determine when graded rings have F-rational or F-regular Veronese subrings. The question regarding F-rational Veronese subrings is easily answered: Let $(R, m, K)$ be a CohenMacaulay graded domain of dimension $d$, with an isolated singularity at $m$. We show that there exists a positive integer $n$ such that the Veronese sub$\operatorname{ring} R^{(n)}$ is F-rational if and only if $\left[H_{m}^{d}(R)\right]_{0}=0$. With regard to F-regular Veronese subrings, we show that if $R$ is a normal ring generated by degree one elements over a field, then either $R$ is F-regular, or else no Veronese 
subring of $R$ is F-regular. This leads us to the question: If $(R, m, K)$ is a normal graded domain, generated by degree one elements, with an isolated singularity at $m$, then under what conditions is $R$ F-regular? It is easily seen that F-regularity forces the $a$-invariant, $a(R)$, to be negative. For rings of dimension two (although not in higher dimensions) this is also a sufficient condition for F-regularity. We construct rings $R$ of dimension $d \geq 3$ with $a(R)=2-d$ which are not F-regular, while if $a(R)<2-d$, Smith has pointed out that $\operatorname{Proj} R$ is a variety of minimal degree, and $R$ is indeed Fregular. We also construct a rich family of F-rational rings of characteristic zero, with isolated singularities, which have no F-regular Veronese subrings.

We would like to point out that although tight closure is primarily a characteristic $p$ notion, it has strong connections with the study of singularities of algebraic varieties over fields of characteristic zero. Specifically, let $R$ be a ring essentially of finite type over a field of characteristic zero. Then $R$ has rational singularities if and only if it is of F-rational type, see [Ha, Sm3]. In the $\mathbb{Q}$-Gorenstein case, we have some even more remarkable connections: F-regular type is equivalent to having log-terminal singularities and F-pure type implies (and is conjectured to be equivalent to) log-canonical singularities, see [Sm5, Wa4].

\section{Preliminaries.}

Let $R$ be a Noetherian ring of characteristic $p>0$. We shall always use the letter $e$ to denote a variable nonnegative integer, and $q$ to denote the $e$ th power of $p$, i.e., $q=p^{e}$. We shall denote by $F$, the Frobenius endomorphism of $R$, and by $F^{e}$, its $e$ th iteration, i.e., $F^{e}(r)=r^{q}$. For an ideal $I=$ $\left(x_{1}, \ldots, x_{n}\right) \subseteq R$, we let $I^{[q]}=\left(x_{1}^{q}, \ldots, x_{n}^{q}\right)$. Note that $F^{e}(I) R=I^{[q]}$, where $q=p^{e}$, as always. Let $S$ denote the ring $R$ viewed as an $R$-algebra via $F^{e}$. Then $S \otimes_{R}$ - is a covariant functor from $R$-modules to $S$-modules, and so is a covariant functor from $R$-modules to $R$-modules! If we consider a map of free modules $R^{n} \rightarrow R^{m}$ given by the matrix $\left(r_{i j}\right)$, applying $F^{e}$ we get a map $R^{n} \rightarrow R^{m}$ given by the matrix $\left(r_{i j}^{q}\right)$. For an $R$-module $M$, note that the $R$-module structure on $F^{e}(M)$ is $r^{\prime}(r \otimes m)=r^{\prime} r \otimes m$, and $r^{\prime} \otimes r m=r^{\prime} r^{q} \otimes m$. For $R$-modules $N \subseteq M$, we use $N_{M}^{[q]}$ to denote $\operatorname{Im}\left(F^{e}(N) \rightarrow F^{e}(M)\right)$.

For a reduced ring $R$ of characteristic $p>0, R^{1 / q}$ shall denote the ring obtained by adjoining all $q$ th roots of elements of $R$. The ring $R$ is said to be $F$-finite if $R^{1 / p}$ is module-finite over $R$. Note that a finitely generated algebra $R$ over a field $K$ is F-finite if and only if $K^{1 / p}$ is a finite field extension of $K$.

We shall denote by $R^{\circ}$ the complement of the union of the minimal primes of $R$. We say $I=\left(x_{1}, \ldots, x_{n}\right) \subseteq R$ is a parameter ideal if the images of $x_{1}, \ldots, x_{n}$ form part of a system of parameters in the local ring $R_{P}$, for every prime ideal $P$ containing $I$. 
Definition 2.1. Let $R$ be a ring of characteristic $p$, and $I$ an ideal of $R$. An element $x$ of $R$, is said to be in $I^{F}$, the Frobenius closure of $I$, if there exists some $q=p^{e}$ such that $x^{q} \in I^{[q]}$.

For $R$-modules $N \subseteq M$ and $u \in M$, we say that $u \in N_{M}^{*}$, the tight closure of $N$ in $M$, if there exists $c \in R^{\circ}$ such that $c u^{q} \in N_{M}^{[q]}$ for all $q=p^{e} \gg 0$. It is worth recording this when $M=R$, and $N=I$ is an ideal of $R$. An element $x$ of $R$ is said to be in $I^{*}$ if there exists $c \in R^{\circ}$ such that $c x^{q} \in I^{[q]}$ for all $q=p^{e} \gg 0$. If $I^{*}=I$ we say that the ideal $I$ is tightly closed.

A ring $R$ is said to be $F$-pure if for all $R$-modules $M$, the Frobenius homomorphism $F: M \rightarrow F(M)$ is injective. A ring $R$ is weakly F-regular if every ideal of $R$ is tightly closed, and is F-regular if every localization is weakly F-regular. Lastly, $R$ is said to be F-rational if every parameter ideal of $R$ is tightly closed.

It is easily verified that $I \subseteq I^{F} \subseteq I^{*}$. Furthermore, $I^{*}$ is always contained in the integral closure of $I$, and is frequently much smaller. A weakly Fregular ring is F-rational as well as F-pure. We next record some useful results.

\section{Theorem 2.2.}

(1) Regular rings are F-regular. A ring which is a direct summand of an $F$-regular ring is itself F-regular.

(2) An F-rational ring $R$ is normal. If, in addition, $R$ is the homomorphic image of a Cohen-Macaulay ring, then it is Cohen-Macaulay.

(3) An F-rational Gorenstein ring is F-regular.

(4) Let $(R, m)$ be a reduced excellent local ring of dimension d and characteristic $p>0$. If $c \in R^{\circ}$ is an element such that $R_{c}$ is F-rational, then there exists a positive integer $N$ such that $c^{N}\left(0_{H_{m}^{d}(R)}^{*}\right)=0$.

(5) Let $R$ be a graded ring. Then $R$ is weakly F-regular if and only if it is F-regular.

Proof. For assertions (1)-(3), see [HH2, Theorem 4.2]. Part (4) is a result of Velez, [Ve], and (5) is [LS, Corollary 4.4].

Remark 2.3. The equivalence of weak F-regularity and F-regularity, in general, is a formidable open question. However in the light of Theorem 2.2 (5) above, we frequently have no reason to distinguish between these notions.

By a graded ring $(R, m, K)$, we shall always mean a ring $R=\oplus_{n \geq 0} R_{n}$ finitely generated over a field $R_{0}=K$. We shall denote by $m=R_{+}$, the homogeneous maximal ideal of $R$. The punctured spectrum of $R$ refers to the set Spec $R-\{m\}$. By a system of parameters for $R$, we shall mean a sequence of homogeneous elements of $R$ whose images form a system of parameters for $R_{m}$. In specific examples involving homomorphic images of polynomial rings, 
lower case letters shall denote the images of the corresponding variables, the variables being denoted by upper case letters.

For conventions regarding graded modules and homomorphisms, we follow $[\mathbf{G W}]$. For a graded $R$-module $M$, we shall denote by $[M]_{i}$, the $i$-th graded piece of $M$.

Definition 2.4. Let $R=\oplus_{i \geq 0} R_{i}$ be a graded ring, and $n$ be a positive integer. We shall denote by $R^{(n)}$, the Veronese subring of $R$ spanned by all elements of $R$ which have degree a multiple of $n$, i.e., $R^{(n)}=\oplus_{i \geq 0} R_{i n}$.

Note that the ring $R^{(n)}$ is a direct summand of $R$ as an $R^{(n)}$-module and that $R$ is integral over $R^{(n)}$. Hence whenever $R$ is Cohen-Macaulay or normal, so is $R^{(n)}$. We record the following result, see [EGA, Lemme 2.1.6] or [Mu, page 282] for a proof.

Lemma 2.5. Let $R$ be a graded ring. Then there exists a positive integer $n$ such that the Veronese subring $R^{(n)}$ is generated over $K$ by forms of equal degree.

Recall that the highest local cohomology module $H_{m}^{d}(R)$ of $R$, where $\operatorname{dim} R=d$, may be identified with $\lim _{\longrightarrow} R /\left(x_{1}^{t}, \ldots, x_{d}^{t}\right)$ where $x_{1}, \ldots, x_{d}$ is a system of parameters for $R$ and the maps are induced by multiplication by $x_{1} \cdots x_{d}$. If $R$ is Cohen-Macaulay, these maps are injective. The $R$-module $H_{m}^{d}(R)$ carries a natural graded structure, namely $\operatorname{deg}\left[r+\left(x_{1}^{t}, \ldots, x_{d}^{t}\right)\right]=$ $\operatorname{deg} r-t \sum_{i=1}^{d} x_{i}$, where $r$ and $x_{i}$ are homogeneous elements of $R$.

Definition 2.6. In the above setting, Goto and Watanabe define the $a$ invariant of $R$ as the highest integer $a(R)=a$ such that $\left[H_{m}^{d}(R)\right]_{a}$ is nonzero.

When $R$ is a ring of characteristic $p$, the Frobenius homomorphism of $R$ gives a natural Frobenius action on $H_{m}^{d}(R)$ where

$$
F:\left[r+\left(x_{1}^{t}, \ldots, x_{d}^{t}\right)\right] \mapsto\left[r^{p}+\left(x_{1}^{p t}, \ldots, x_{d}^{p t}\right)\right], \quad \text { see }[\mathbf{F W}] \text { or }[\mathbf{S m 2}] .
$$

For a graded $R$-module $M$, define $M^{(n)}=\oplus_{i \in \mathbb{Z}}[M]_{i n}$. With this notation, it follows from [GW, Theorem 3.1.1] that

$$
H_{m_{R^{(n)}}}^{d}\left(R^{(n)}\right) \cong\left(H_{m}^{d}(R)\right)^{(n)} .
$$

The following theorem, [HH3, Theorem 7.12], indicates the importance of the $a$-invariant in the study of graded F-rational rings.

Theorem 2.7. A graded Cohen-Macaulay normal ring $R$ over a field of prime characteristic $p$ is F-rational if and only if $a(R)<0$ and the ideal generated by some homogeneous system of parameters for $R$ is Frobenius closed. 


\section{F-rationality of Veronese subrings.}

The following proposition, well-known to the experts, addresses the existence of F-rational Veronese subrings.

Proposition 3.1. Let $R$ be a graded Cohen-Macaulay domain of dimension $d$, which is locally F-rational on the punctured spectrum $\operatorname{Spec} R-m$. (This is satisfied, in particular, if $R$ has an isolated singularity.) Then $\left[H_{m}^{d}(R)\right]_{0}=0$ if and only if the Veronese subring $R^{(n)}$ is F-rational for all integers $n \gg 0$. In particular if $a(R)<0$, then $R^{(n)}$ is F-rational for all integers $n \gg 0$.

Proof. Note that we have $\left[H_{m}^{d}(R)\right]_{0} \subseteq 0_{H_{m}^{d}(R)}^{*}$, since for $z \in\left[H_{m}^{d}(R)\right]_{0}$ we get $c z^{q}=0$ for all $q=p^{e}$, when $c \in m$ is of a sufficiently large degree. Consequently if $R^{(n)}$ is F-rational for some $n$, we must have $a\left(R^{(n)}\right)<0$, but then $\left[H_{m}^{d}(R)\right]_{0}=0$.

For the converse first note that since $R$ is F-rational on the punctured spectrum, Theorem $2.2(4)$ says that $0_{H_{m}^{d}(R)}^{*}$ must be killed by a power of the maximal ideal $m$, and so is of finite length. As $\left[H_{m}^{d}(R)\right]_{0}=0$, for large positive integers $n$ we see that $H_{m^{\prime}}^{d}\left(R^{(n)}\right) \cong\left(H_{m}^{d}(R)\right)^{(n)}$ contains no nonzero element of $0_{H_{m}^{d}(R)}^{*}$ where $m^{\prime}$ denotes the homogeneous maximal ideal of $R^{(n)}$. If $u \in 0_{H_{m^{\prime}}^{d}\left(R^{(n)}\right)}^{*}$ then $u \in 0_{H_{m}^{d}(R)}^{*} \cap H_{m^{\prime}}^{d}\left(R^{(n)}\right)$ and so $u=0$. Hence $R^{(n)}$ is F-rational for $n \gg 0$.

Example 3.2. Let $R=K[X, Y, Z] /\left(X^{2}+Y^{3}+Z^{5}\right)$ where $K$ is a field of prime characteristic $p$. We make this a graded ring by setting the weights of $x, y$ and $z$ to be 15, 10 and 6 respectively. We determine the positive integers $n$ for which the Veronese subring $R^{(n)}$ is F-rational. This shall, of course, depend on the characteristic $p$ of $R$.

First note that $a(R)=-1$ with this grading. If $p \geq 7$, it is easy to verify that the ring $R$ is F-regular. Consequently every Veronese subring of $R$, being a direct summand of $R$, is also F-regular. For $p=2,3$ or $5, x^{p} \in\left(y^{p}, z^{p}\right)$, and so $R$ is not F-rational. It is also easily checked that the action of the Frobenius on $H_{m}^{2}(R)$ is injective in degree $\leq-2$ with the one exception of $p=2$ where elements in degree -7 are mapped to zero under the action of the Frobenius, specifically $F\left(x y^{-1} z^{-2}\right)=0$ in $H_{m}^{2}(R)$. Recall that $H_{m_{R^{(n)}}}^{2}\left(R^{(n)}\right)$ is generated by elements of $H_{m}^{2}(R)$ whose degree is a multiple of $n$. Consequently for $n \geq 2$ the action of the Frobenius on $H_{m_{R^{(n)}}}^{2}\left(R^{(n)}\right)$ is injective, with the one exception. Using the arguments in the proof of the above proposition, we see that $R^{(n)}$ is F-rational for all $n \geq 2$, excluding the case when $p=2$ and $n=7$. 


\section{Rational coefficient Weil divisors.}

We review some notation and results from [De], [Wa1] and [Wa3], as well as make a few observations which we shall find useful later in our study.

Definition 4.1. By a rational coefficient Weil divisor (or a $\mathbb{Q}$-divisor) on a normal projective variety $X$, we mean a $\mathbb{Q}$-linear combination of codimension one irreducible subvarieties of $X$. For $D=\sum n_{i} V_{i}$ with $n_{i} \in \mathbb{Q}$, we set $[D]=\sum\left[n_{i}\right] V_{i}$, where $[n]$ denotes the greatest integer less than or equal to $n$, and define $\mathcal{O}_{X}(D)=\mathcal{O}_{X}([D])$.

Let $D=\sum\left(p_{i} / q_{i}\right) V_{i}$ where the integers $p_{i}$ and $q_{i}$ are relatively prime and $q_{i}>0$. We define $D^{\prime}=\sum\left(\left(q_{i}-1\right) / q_{i}\right) V_{i}$ to be the fractional part of $D$. Note that with this definition of $D^{\prime}$ we have $-[-n D]=\left[n D+D^{\prime}\right]$ for any integer $n$.

Given an ample $\mathbb{Q}$-divisor $D$ (i.e., such that $N D$ is an ample Cartier divisor for some $N \in \mathbb{N}$ ), we construct the generalized section ring:

$$
R=R(X, D)=\oplus_{n \geq 0} H^{0}\left(X, \mathcal{O}_{X}(n D)\right) T^{n} \subseteq K(X)[T] .
$$

With this notation, Demazure's result $([\mathbf{D e}, 3.5])$ is:

Theorem 4.2. Let $R=\oplus_{n \geq 0} R_{n}$ be a graded normal ring. Then there exists an ample $\mathbb{Q}$-divisor $D$ on $X=\operatorname{Proj} R$ such that

$$
R=\oplus_{n \geq 0} H^{0}\left(X, \mathcal{O}_{X}(n D)\right) T^{n} \subseteq K(X)[T],
$$

where $T$ is a homogeneous element of degree one in the quotient field of $R$.

Example 4.3. Take the $\mathbb{Q}$-divisor

$$
D=(-1 / 2) V(S)+(1 / 3) V(T)+(1 / 5) V(S+T)
$$

on $\mathbb{P}^{1}=\operatorname{Proj} K[S, T]$ where $V(S)$, e.g., denotes the irreducible subvariety defined by the vanishing of $S$. Fix $T$ as the degree one element. Then

$$
\begin{gathered}
R=\oplus_{n \geq 0} H^{0}\left(\mathbb{P}^{1}, \mathcal{O}_{\mathbb{P}^{1}}(n D)\right) T^{n}=K[X, Y, Z] /\left(X^{2}+Y^{3}+Z^{5}\right), \quad \text { where } \\
X=\left(S^{8} T^{10}\right) /(S+T)^{3}, Y=\left(S^{5} T^{7}\right) /(S+T)^{2} \text {, and } Z=\left(-S^{3} T^{4}\right) /(S+T) .
\end{gathered}
$$

Remark 4.4. Let $R=R(X, D)$ be as above. Then the Veronese subring $R^{(n)}$ is given by $R^{(n)} \cong R(X, n D)$. For a rational function $f \in K(X)$ we have an isomorphism $R(X, D) \cong R(X, \operatorname{div}(f)+D)$. If $R$ is generated over $K$ by its elements of degree one, we have $R=R(X,[D])$. Note that $[D]$ is a Weil divisor, i.e., has integer coefficients.

\section{Results in dimension two.}

In the following theorem, we summarize some familiar results about graded rings of dimension two. 
Theorem 5.1. Let $R$ be a graded normal ring of dimension two, which is generated by degree one elements over an algebraically closed field. Then the following statements are equivalent:

(1) $R$ is isomorphic to a Veronese subring of a polynomial ring in two variables.

(2) $R$ is F-regular.

(3) $R$ is F-rational.

(4) $R$ has a negative a-invariant.

Proof. The implications $(1) \Rightarrow(2) \Rightarrow(3) \Rightarrow(4)$ follow easily. For $(4) \Rightarrow(1)$ note that $X=\operatorname{Proj} R$ is a nonsingular projective curve. Since $\left[H_{m}^{2}(R)\right]_{0}=0$, we have $H^{1}\left(X, \mathcal{O}_{X}\right)=0$ and so $X$ is of genus zero, i.e., $\mathbb{P}^{1}$. Consequently $R \cong R\left(\mathbb{P}^{1}, D\right)$ where $D$ is a Weil divisor on $\mathbb{P}^{1}$. Hence $D$ is linearly equivalent to $\mathcal{O}(m)$ for some $m \in \mathbb{N}$ and $R \cong R\left(\mathbb{P}^{1}, \mathcal{O}(m)\right) \cong\left(K\left[X_{0}, X_{1}\right]\right)^{(m)}$.

As an easy consequence of the above, we have:

Theorem 5.2. Let $R$ be a graded domain of dimension two, with an isolated singularity, which is finitely generated over an algebraically closed field. If $a(R)<0$, there exists a positive integer $n$ such that $R^{(n)}$ is isomorphic to a Veronese subring of a polynomial ring in two variables over $K$. In particular, some Veronese subring of $R$ is F-regular.

Proof. Note that $R$ is excellent and so $R^{\prime}$, the integral closure $R$ in its fraction field, is module-finite over $R$. Since $R$ has an isolated singularity, the conductor (i.e., the largest common ideal of $R$ and $R^{\prime}$ ) is primary to the maximal ideal of $R^{\prime}$, by which $R_{i}=R_{i}^{\prime}$ for all $i \gg 0$. We may therefore choose a positive integer $k$ such that $R^{(k)}$ is normal, and then choose an appropriate multiple $n$ of $k$, by Lemma 2.5, such that $R^{(n)}$ is generated by elements of equal degree. We are now in a position to apply the above theorem to conclude that $R^{(n)}$ is isomorphic to a Veronese subring of a polynomial ring in two variables.

Example 5.3. Let $S=K[X, Y, Z] /\left(X^{3}-Y Z(Y+Z)\right)$ where $K$ is a field of characteristic $p \equiv 1(\bmod 3)$ and consider the subring

$$
R=K\left[X, Y^{3}, Y^{2} Z, Y Z^{2}, Z^{3}\right] /\left(X^{3}-Y Z(Y+Z)\right) .
$$

It is proved in [HH3] that $R$ is F-rational but not F-regular, see also [Wa3]. Since $R^{(3)}$ is generated by elements of equal degree, it must be isomorphic to a Veronese subring of a polynomial ring by Theorem 5.1. Indeed,

$$
R^{(3)}=K\left[Y^{3}, Y^{2} Z, Y Z^{2}, Z^{3}\right] .
$$

Example 5.4. Let $R=K\left[t, t^{4} x, t^{4} x^{-1}, t^{4}(x+1)^{-1}\right]$ where $K$ is a field of prime characteristic $p$. This is one of the examples in [Wa2] of rings which 
are F-rational but not F-pure; for a different proof see [HH3]. By mapping a polynomial ring onto it, we may write $R$ as

$$
R=K[T, U, V, W] /\left(T^{8}-U V, T^{4}(V-W)-V W, U(V-W)-T^{4} W\right) .
$$

This is graded by setting the weights of $t, u, v$ and $w$ to be $1,4,4$ and 4 respectively. Note that

$$
R^{(4)}=K[S, U, V, W] /\left(S^{2}-U V, S(V-W)-V W, U(V-W)-S W\right)
$$

where we relabel $T^{4}$ as $S$. Then $R^{(4)}$ is generated by elements of equal degree, and is isomorphic to $K\left[X^{3}, X^{2} Y, X Y^{2}, Y^{3}\right]$ by setting $S=X Y(X-Y)$, $U=X Y^{2}, V=X(X-Y)^{2}$, and $W=Y(X-Y)^{2}$.

By Theorem 5.2 we know that a graded normal ring $R$ of dimension two over an algebraically closed field has a Veronese subring $R^{(n)}$ which is Fregular. We next show that if $R$ is a hypersurface, there exists $n$ such that $R^{(n)}$ is actually an F-regular hypersurface.

Theorem 5.5. Let $R$ be a graded normal hypersurface of dimension two with $a(R)<0$. Then there exists a positive integer $n$ such that the Veronese subring $R^{(n)}$ is an F-regular hypersurface.

Proof. Let $R=K[X, Y, Z] /(f)$ where $x, y$ and $z$ have weights $m, n$ and $r$ respectively. We may assume without any loss of generality that $m, n$ and $r$ have no common factor. If $d=\operatorname{gcd}(m, n)$, then by our assumption $d$ and $r$ are relatively prime. Therefore $f$ must be a polynomial in $x, y$ and $z^{d}$. Consequently $R^{(n)}$ is again a hypersurface, and satisfies all the initial hypotheses, and so we may assume that $R$ satisfies the extra hypothesis that $m, n$ and $r$ are pairwise relatively prime. Assume further that $m \geq n \geq r$. We consider the two cases: a) $n=1$ and $r=1$, and b) $m>n>r$. Note that it suffices to show that $R$ is F-rational, since it is indeed a hypersurface.

We first eliminate the case (\#) when $f$ is of the form $X H(Y, Z)+G(Y, Z)$. We may take a system of parameters of $R$ of the form $x, t$ where $t$ is the image in $R$ of a polynomial $T \in K[X, Y, Z]$ involving only $Y$ and $Z$. If $R$ is not F-rational, then since $a(R)<0,(x, t)$ cannot be F-pure. Hence for some $q=p^{e}$, we have $s^{q} \in\left(x^{q}, t^{q}\right)$ while $s \notin(x, t)$. Again, we may assume that $s$ is the image in $R$ of a polynomial $S \in K[X, Y, Z]$ involving only $Y$ and $Z$. This means that in $K[X, Y, Z]$, we have $S^{q} \in\left(X^{q}, T^{q}, X H+G\right)$ but then $S^{q} \in\left(T^{q}, G^{q}\right)$ and so $S \in(T, G)$ in $K[X, Y, Z]$, giving us the contradiction $s \in(x, t)$.

a) We have $a(R)=\operatorname{deg} f-(m+n+r)<0$, and so $\operatorname{deg} f<m+2$ since $n=r=1$. This forces $f$ to be of the form (\#).

b) Since $a(R)=\operatorname{deg} f-(m+n+r)<0$, we have $\operatorname{deg} f<m+n+r<3 m$. Hence up to a scalar multiple, $f$ is of the form $X H(Y, Z)+G(Y, Z)$ or $X^{2}+G(Y, Z)$. Note that the first case has already been handled. 
Now suppose $f=X^{2}+G(Y, Z)$. Then $\operatorname{deg} f=2 m<m+n+r$ and so $3<m<n+r$, consequently $G$ cannot involve a term of the form $Y^{2} Z^{l}$ where $l \geq 2$. If $G$ has a term $Y^{k}$, then $2 m=k n$ and so $n=1$ or 2 . Since $n>r$, we can only have $n=2$ and $r=1$, but this too is impossible. Hence $f$ can only be of the form $f=X^{2}+a Z^{k}+b Y Z^{l}+c Y^{2} Z$ where $a, b$ and $c$ are scalars. $R$ is normal, and so $c$ must be non-zero since $l \geq 2$ and $k \geq 2$. It follows that $2 m=2 n+r$. If $a$ is non-zero, $2 m=r k$ and since $r$ is even, we can only have $r=2$. But then $m=n+1$, and so $r$ divides either $m$ or $n$, a contradiction. Hence $a=0$, and so $f=X^{2}+b Y Z^{l}+c Y^{2} Z$. If $b$ were non-zero, then we would have $n+r l=2 n+r$, i.e., $n=r(l-1)$, which forces $r=1$. However we know $r$ to be even, and so $b=0$. We are left with $f=X^{2}+c Y^{2} Z$ but this is ruled out since $R$ is normal.

\section{F-regular Veronese subrings.}

We begin by recalling a theorem of Watanabe, [Wa3, Theorem 3.4]:

Theorem 6.1. Let $D_{1}$ and $D_{2}$ be ample $\mathbb{Q}$-divisors on a normal projective variety $X$. If the fractional parts $D_{1}^{\prime}$ and $D_{2}^{\prime}$ are equal, then the ring $R\left(X, D_{1}\right)$ is F-regular (F-pure) if and only if the ring $R\left(X, D_{2}\right)$ is F-regular (F-pure).

A complete proof of the theorem, as stated above, relies on the characterization of strong F-regularity in terms of the tight closure of the zero submodule of the injective hull of the residue field, [Sm1, Proposition 7.1.2], as well as the results of $[\mathbf{L S}]$.

Corollary 6.2. Let $R$ be a graded normal ring which is generated by degree one elements over a field. Then either $R$ is F-regular (F-pure), or else no Veronese subring of $R$ is F-regular (F-pure).

Proof. Since $R$ is generated by its elements of degree one, we have $R=$ $R(X, D)$, where $D$ is a Weil divisor, i.e., has $D^{\prime}=0$. Also, $(n D)^{\prime}=0$ where $n$ is any positive integer. By the above Theorem, $R=R(X, D)$ is F-regular (F-pure) if and only if $R^{(n)} \cong R(X, n D)$ is F-regular (F-pure).

As an application of this result, we now construct a family of rings with negative $a$-invariants, which have no F-pure Veronese subrings. This shows that a result corresponding to Theorem 5.2 is no longer true in higher dimensions.

Example 6.3. Let $R=K\left[X_{0}, \ldots, X_{d}\right] /\left(X_{0}^{3}+\cdots+X_{d}^{3}\right)$ with $d \geq 3$, where $K$ is a field of characteristic 2 . It is readily seen that $x_{0}^{2} \in\left(x_{1}, \ldots, x_{d}\right)^{*}$, since $x_{0}^{4} \in\left(x_{1}, \ldots, x_{d}\right)^{[2]}$. Hence $R$ is not F-pure, and since it is generated by elements of degree one, Corollary 6.2 shows that $R$ has no F-regular or F-pure Veronese subrings. Note that $a(R)=2-d<0$. 
We can also see that $R^{(n)}$ is not F-pure (for any $n>0$ ) by showing that the element $x_{0}^{d}\left(x_{1} \cdots x_{d}\right)^{n-1}$ is in the Frobenius closure of the ideal

$$
\left(x_{0}^{d-2} x_{1}^{n} x_{2}^{n-1} \cdots x_{d-1}^{n-1}, \quad x_{0}^{d-2} x_{2}^{n} x_{3}^{n-1} \cdots x_{d}^{n-1}, \ldots, \quad x_{0}^{d-2} x_{d}^{n} x_{1}^{n-1} \cdots x_{d-2}^{n-1}\right),
$$

although not in the ideal itself.

For all $n \geq 2$, the ring $R^{(n)}$ is an example of a graded ring generated by degree one elements (with an isolated singularity and a negative $a$-invariant) which is F-rational but not F-pure.

Remark 6.4. The examples above are not completely satisfactory as they are not valid in the characteristic zero setting: in fact, for $d \geq 3$, the ring $R=\mathbb{Q}\left[X_{0}, \ldots, X_{d}\right] /\left(X_{0}^{3}+\cdots+X_{d}^{3}\right)$ is of F-regular type. Characteristic zero examples turn out to be much more subtle, and we construct these in the next section.

We again return to the ring $R=K[X, Y, Z] /\left(X^{2}+Y^{3}+Z^{5}\right)$, and this time determine its F-regular and F-pure Veronese subrings.

Example 6.5. Let $R=K[X, Y, Z] /\left(X^{2}+Y^{3}+Z^{5}\right)$ where $K$ is a field of prime characteristic $p$, and the grading is as before. For $p \geq 7$ the ring $R$ is F-regular, and therefore so is any Veronese subring $R^{(n)}$. We now determine when $R^{(n)}$ is F-regular assuming $p$ is either 2,3 or 5 .

Note that the Veronese subrings $R^{(2)}, R^{(3)}$ and $R^{(5)}$ are in fact polynomial rings. Therefore when $n$ is divisible by one of 2,3 or $5, R^{(n)}$ is a direct summand of a polynomial ring, and so is F-regular. We show that these are the only instances when $R^{(n)}$ is F-regular, or even F-pure.

Recall from Example 4.3 that $R=R(X, D)$ where $X=\operatorname{Proj} K[S, T]$ and $D=(-1 / 2) V(S)+(1 / 3) V(T)+(1 / 5) V(S+T)$. If $n$ is relatively prime to 30, the $\mathbb{Q}$-divisor $n D$ has the same fractional part as $D$, and so $R^{(n)} \cong R(X, n D)$ is not F-pure or F-regular by Theorem 6.1.

We can also construct explicit instances of Frobenius closure to illustrate why $R^{(n)}$ is not F-pure when $n$ is relatively prime to 30 . Since $n$ is relatively prime to the weight of $y$, the ring $R^{(n)}$ has a unique monomial of the form $x y^{l}$ with $0<l<n$. Similarly there is a unique integer $m$ with $0 \leq m<n$ such that $y^{l+1} z^{m} \in R^{(n)}$, and a unique integer $r$ with $0<r<n$ such that $x^{r} z \in R^{(n)}$. We claim that

$$
\begin{aligned}
& x^{r+1} y^{r l+l} z^{d} \in\left(x^{r} y^{r l+l+1} z^{m}, x^{r} z^{d+1}\right)^{F}, \text { and } \\
& x^{r+1} y^{r l+l} z^{d} \notin\left(x^{r} y^{r l+l+1} z^{m}, x^{r} z^{d+1}\right) .
\end{aligned}
$$

The second statement is true in $R$ and so also in $R^{(n)}$, while the first assertion follows from

$$
\left(x^{r+1} y^{r l+l} z^{d}\right)^{p} \in\left(\left(x^{r} y^{r l+l+1} z^{m}\right)^{p},\left(x^{r} z^{d+1}\right)^{p}\right) \text { for } p=2,3 \text { or } 5 .
$$


Example 6.6. We saw that the F-purity and F-regularity of a ring $R=$ $R(X, D)$ depend only on the fractional part $D^{\prime}$ of the $\mathbb{Q}$-divisor $D$. This is by no means true of F-rationality and F-injectivity (i.e., the injectivity of the Frobenius action on the highest local cohomology module). As an example of this, consider the $\mathbb{Q}$-divisors on Proj $K[S, T]$

$$
\begin{aligned}
& E=(1 / 2) V(S)+(1 / 3) V(T)+(1 / 5) V(S+T) \text { and } \\
& D=(-1 / 2) V(S)+(1 / 3) V(T)+(1 / 5) V(S+T),
\end{aligned}
$$

which have the same fractional part. Then

$$
S=\oplus_{n \geq 0} H^{0}\left(X, \mathcal{O}_{X}(n E)\right) T^{n} \cong K[A, B, C, T] / I
$$

where $I=\left(A B-T^{5}, B C+C T^{3}-B T^{5}, A C+C T^{2}-A B T^{2}\right)$ and $A=T^{3} / S$, $B=S T^{2}$ and $C=S T^{5} /(S+T)$. If the characteristic of $K$ is 2,3 or 5 , the ring $R=R(X, D)=K[X, Y, Z] /\left(X^{2}+Y^{3}+Z^{5}\right)$ is not F-rational (or F-injective) as we saw in Example 3.2. We claim that the ring $S$ is however Frational. To see this note that $a(S)<0$, and so it suffices by Theorem 2.7 to verify that the ideal $I$ generated by the homogeneous system of parameters $t, a^{15}+b^{10}+c^{6}$ is Frobenius closed. However this is easily verified: The ring $S / t S \cong K[A, B, C] /(A B, B C, C A)$ is F-pure since the ideal $(A B, B C, C A)$ is generated by square free monomials, see [HR2, Proposition 5.38].

Remark 6.7. Let $R$ be a Cohen-Macaulay ring with an isolated singularity, which is generated by degree one elements over an algebraically closed field. For a two dimensional ring $R$, a negative $a$-invariant forces $R$ to be F-regular, although for rings of higher dimensions this is no longer true: In Example 6.3 we constructed rings $R$ of dimension $d>3$, with $a(R)=2-d$, which were not F-regular. Smith has pointed out that if $R$ satisfies the stronger condition that $a(R) \leq 1-d$, then Proj $R$ is a variety of minimal degree. These are completely classified (see, for example, $[\mathbf{E H}]$ ) and it is easily verified that in this case $R$ is F-regular, see [Sm4, Remark 4.3.1].

\section{The case of characteristic zero.}

Hochster and Huneke have defined analogous notions of tight closure for rings essentially of finite type over a field of characteristic zero, see $[\mathbf{H H} \mathbf{1}$, HH4]. However we can also define notions corresponding to F-regularity, F-purity, and F-rationality in characteristic zero, without using a closure operation.

Consider the ring $R=K\left[X_{1}, \ldots, X_{n}\right] / I$ where $K$ is a field of characteristic zero. Choose a finitely generated $\mathbb{Z}$-algebra $A$ such that $R_{A}=$ $A\left[X_{1}, \ldots, X_{n}\right] / I_{A}$ is a free $A$-algebra, with $R \cong R_{A} \otimes_{A} K$. Note that the fibers of the homomorphism $A \rightarrow R_{A}$ over maximal ideals of $A$ are finitely generated algebras over fields of prime characteristic. 
Definition 7.1. Let $R$ be a ring finitely generated over a field of characteristic zero. Then $R$ is said to be of F-regular type if there exists a finitely generated $\mathbb{Z}$-algebra $A \subseteq K$ and a finitely generated $A$-algebra $R_{A}$ such that $R \cong R_{A} \otimes_{A} K$, and for all maximal ideals $\mu$ in a Zariski dense subset of Spec $A$, the fiber rings $R_{A} \otimes_{A} A / \mu$ are F-regular.

Similarly, $R$ is said to be of $F$-pure type if for all maximal ideals $\mu$ in a Zariski dense subset of $\operatorname{Spec} A$, the fiber rings $R_{A} \otimes_{A} A / \mu$ are F-pure.

Remark 7.2. Some authors use the term F-pure type (F-regular type) to mean that $R_{A} \otimes_{A} A / \mu$ is F-pure (F-regular) for all maximal ideals $\mu$ in a Zariski dense open subset of $\operatorname{Spec} A$.

All our positive results towards the existence of F-rational and F-regular Veronese subrings in prime characteristic do have corresponding statements in the characteristic zero situation. However we have so far not exhibited a normal Cohen-Macaulay ring, generated by degree one elements over a field of characteristic zero, which has an isolated singularity and a negative $a$-invariant but is not of F-regular type. N. Hara has pointed out to us a geometric argument for the existence of such rings using a blow-up of $\mathbb{P}^{2}$ at nine points. In this section, we construct a large family of explicit examples of such rings of dimension $d \geq 3$.

Example 7.3. Take two relatively prime homogeneous polynomials $F$ and $G$ of degree $d$ in the ring $\mathbb{Z}\left[X_{1}, \ldots, X_{k}\right]$, where $k \geq 3$, such that $G$ is monic in $X_{k}$ and the monomial $X_{k}^{d}$ does not occur in $F$. Using $F$ and $G$, construct the hypersurface $S=\mathbb{Q}\left[S, T, X_{1}, \ldots, X_{k}\right] /(S F-T G)$ and let $R$ be the subring of $S$ generated by the elements $s x_{1}, \ldots, s x_{k}, t x_{1}, \ldots, t x_{k}$.

For suitably general choices of the polynomials $F$ and $G$ of degree $d=k$ the ring $R$ has only isolated singularities, and we show that it is CohenMacaulay with $a(R)=-1$, and is not of F-regular type. For an explicit example, take $k=3, F=X_{1} X_{2} X_{3}$ and $G=X_{1}^{3}+X_{2}^{3}+X_{3}^{3}$.

We shall prove that $R$ is Cohen-Macaulay whenever $d \leq k$. We first show that the Hilbert polynomial multiplicity of $R$ is $d(k-1)+1$, and then construct a system of parameters such that the ring obtained by killing this system of parameters has length $d(k-1)+1$.

We construct a basis for the vector space generated by the monomials of degree $n \gg 0, s^{i} t^{n-i} x_{1}^{j_{1}} x_{2}^{j_{2}} \cdots x_{k}^{j_{k}}$, where the $j_{r}$ are nonnegative integers which add up to $n$. The relations permit us to express $t x_{k}^{d}$ in terms of other monomials. Let $\left[u_{1}, \ldots, u_{m}\right]^{i}$ denote the set $\mathcal{S}$ of monomials of degree $i$ in $u_{1}, \ldots, u_{m}$, and for two such sets, let $\mathcal{S} \cdot T$ denote the product of all possible pairs from $\mathcal{S}$ and $\mathcal{T}$. In this notation, for $n \gg 0$, the following monomials 
constitute a basis for $R_{n}$ :

$$
\begin{aligned}
& {[s, t]^{n} \cdot\left[x_{1}, \ldots, x_{k-1}\right]^{n},} \\
& {[s, t]^{n} \cdot\left[x_{1}, \ldots, x_{k-1}\right]^{n-1} \cdot\left[x_{k}\right],} \\
& \ldots \\
& {[s, t]^{n} \cdot\left[x_{1}, \ldots, x_{k-1}\right]^{n-d+1} \cdot\left[x_{k}\right]^{d-1},} \\
& {[s]^{n} \cdot\left[x_{1}, \ldots, x_{k}\right]^{n-d} \cdot\left[x_{k}\right]^{d} .}
\end{aligned}
$$

Consequently for large $n$ the vector space dimension of $R_{n}$ is

$$
(n+1)\left\{\left(\begin{array}{c}
n+k-2 \\
k-2
\end{array}\right)+\cdots+\left(\begin{array}{c}
n-d+1+k-2 \\
k-2
\end{array}\right)\right\}+\left(\begin{array}{c}
n-d+k-1 \\
k-1
\end{array}\right) .
$$

As a polynomial in $n$, the leading term of this expression is

$$
n\left\{\frac{n^{k-2}}{(k-2) !}+\cdots+\frac{n^{k-2}}{(k-2) !}\right\}+\frac{n^{k-1}}{(k-1) !}=\frac{n^{k-1}(d(k-1)+1)}{(k-1) !},
$$

and so the Hilbert polynomial multiplicity of $R$ is $d(k-1)+1$.

The sequence of elements $s x_{1}, s x_{2}-t x_{1}, s x_{3}-t x_{2}, \ldots, s x_{k}-t x_{k-1}$ is a system of parameters for $R$. Since we have already verified that the Hilbert polynomial multiplicity of $R$ is $d(k-1)+1$, to prove that $R$ is Cohen-Macaulay when $d \leq k$, it suffices to show that the length of the ring $T$ obtained by killing this system of parameters is at most $d(k-1)+1$.

Relabel the generators of $T$ as $a_{2}=s x_{2}, a_{3}=s x_{3}, \ldots, a_{k}=s x_{k}, a_{k+1}=$ $t x_{k}$. Note that the relations amongst the $a_{i}$ include the size two minors of the matrix

$$
\left(\begin{array}{ccccc}
0 & a_{2} & \ldots & a_{k-1} & a_{k} \\
a_{2} & a_{3} & \ldots & a_{k} & a_{k+1}
\end{array}\right) .
$$

Consequently a generating set for $[T]_{<d}$ is given by

$$
\begin{aligned}
\operatorname{deg} 0 & : 1, \\
\operatorname{deg} 1 & : a_{2}, a_{3}, \ldots, a_{k+1}, \\
\operatorname{deg} 2 & : a_{2} a_{k+1}, a_{3} a_{k+1}, \ldots, a_{k+1}^{2}, \\
\operatorname{deg} 3 & : a_{2} a_{k+1}^{2}, a_{3} a_{k+1}^{2}, \ldots, a_{k+1}^{3}, \\
& \ldots \\
\operatorname{deg} d-1 & : a_{2} a_{k+1}^{d-2}, a_{3} a_{k+1}^{d-2}, \ldots, a_{k+1}^{d-1} .
\end{aligned}
$$

In degree $d$ the ring $T$ has $d$ additional independent relations coming from the equations $s^{i} t^{d-i} f-s^{i-1} t^{d-i+1} g$, for $1 \leq i \leq d$. Consequently we need $k-d$ generators for the degree $d$ piece of $T$, and one can check that there are no nonzero elements in degree $d+1$. Hence the length of $T$ is bounded by $d(k-1)+1$, and this completes the proof that $R$ is Cohen-Macaulay.

It only remains to show that $R$ is not of F-regular type when $k \leq d$. Consider the fiber $A$ of the map $\mathbb{Z} \rightarrow R_{\mathbb{Z}}$ over an arbitrary closed point 
$p \mathbb{Z}$. Then $A$ is a finitely generated algebra over the finite field $\mathbb{Z} / p \mathbb{Z}$, and it suffices to show that $A$ is not F-regular. Take the ideal

$$
I=\left(s x_{1}, s x_{2}, \ldots, s x_{k-1}, t x_{1}, t x_{2}, \ldots, t x_{k-1}\right) A .
$$

It is easily verified that $\left(t x_{k}\right)^{d-1} \notin I$, and we show that $\left(t x_{k}\right)^{d-1} \in I^{*}$.

To see $\left(t x_{k}\right)^{d-1} \in I^{*}$ it suffices to check that $\alpha_{q}=\left(t x_{k}\right)^{(d-1)(q+1)} \in I^{[q]}$. Using the relation $t^{d} g-t^{d-1} s f$ where $1 \leq i \leq d$, we may rewrite $\alpha_{q}$ with lower powers of $x_{k}$ occurring in the expressions involved. We can proceed in this manner till we are left with terms which involve powers of $x_{k}$ not greater then $d-1$. Hence $\alpha_{q}$ is a sum of terms which are multiples of

$$
s^{i} t^{q(d-1)-i} x_{1}^{j_{1}} x_{2}^{j_{2}} \cdots x_{k-1}^{j_{k-1}}, \quad \text { where } i \leq q(d-1), \quad \text { and } \sum_{r=1}^{k-1} j_{r}=q(d-1) \text {. }
$$

If $\alpha_{q} \notin I^{[q]}$, then $j_{r}<q$ for $1 \leq r \leq k-1$. However on summing these inequalities we get $q(d-1)<q(k-1)$, a contradiction.

Remark 7.4. Consider the polynomial ring $K\left[X_{1}, \ldots, X_{k}\right]$ where $k \geq 3$. It is worth noting that the ring $R$, as above, is isomorphic to a subring of $K\left[X_{1}, \ldots, X_{k}\right]$,

$$
R=K\left[X_{1} F, X_{2} F, \ldots, X_{k} F, X_{1} G, X_{2} G, \ldots, X_{k} G\right] .
$$

We can show that $R$ is Cohen-Macaulay precisely when the degree $d$ of $F$ and $G$ is less than or equal to $k$. It would certainly be interesting to explore generalizations of this construction.

Acknowledgments. The author wishes to thank Melvin Hochster and Karen Smith for many valuable discussions.

\section{References}

[De] M. Demazure, Anneaux gradués normaux, in: Lẽ Dûng Tráng (ed.) Introduction a la theorie des singularitiés II, Hermann, Paris, (1988), 35-68.

[EH] D. Eisenbud and J. Harris, On varieties of minimal degree (A centennial account), Proc. Sympos. Pure Math., 46 (1987), 3-13.

[FW] R. Fedder and K.-i. Watanabe, A characterization of F-regularity in terms of Fpurity, in: Commutative Algebra, Proc. Microprogram, June 15-July 12, 1987; Math. Sci. Res. Inst. Publ., 15, Springer-Verlag, New York, Berlin, Heidelberg, London, Paris, Tokyo, (1989), 227-245.

[GW] S. Goto and K.-i. Watanabe, On graded rings, I, J. Math. Soc. Japan, 30 (1978), 179-213.

[EGA] A. Grothendieck and J. Dieudonne, Élements de géométrie algébrique; II Étude globale élémentaire de quelques classes de morphismes, Inst. Hautes Études Sci. Publ. Math., 8 (1961), 5-222.

[Ha] N. Hara, A characterisation of rational singularities in terms of injectivity of Frobenius maps, Amer. J. Math., 120 (1998), 981-996. 
[HH1] M. Hochster and C. Huneke, Tight closure, invariant theory, and the BriançonSkoda theorem, J. Amer. Math. Soc., 3 (1990), 31-116.

[HH2] , F-regularity, test elements, and smooth base change, Trans. Amer. Math. Soc., 346 (1994), 1-62.

[HH3] - Tight closure of parameter ideals and splitting in module-finite extensions, J. Algebraic Geom., 3 (1994), 599-670.

[HH4] , Tight closure in equal characteristic zero, in preparation.

[HR1] M. Hochster and J. Roberts, Rings of invariants of reductive groups acting on regular rings are Cohen-Macaulay, Adv. Math., 13 (1974), 115-175.

[HR2] _ The purity of the Frobenius and local cohomology, Adv. Math., 21 (1976), $117-172$.

[LS] G. Lyubeznik and K.E. Smith, Strong and weak F-regularity are equivalent for graded rings, preprint.

[Mu] D. Mumford, The red book of varieties and schemes, Lecture notes in Math., 1358, Springer-Verlag, Berlin, New York, 1988.

[Sm1] K.E. Smith, Tight closure of parameter ideals and F-rationality, Thesis, Univ. of Michigan, 1993.

[Sm2] _ Tight closure of parameter ideals, Invent. Math., 115 (1994), 41-60.

[Sm3] _ F-rational rings have rational singularities, Amer. J. Math., 119 (1997), 159-180.

[Sm4] _ Tight closure in graded rings, J. Math. Kyoto Univ., 37 (1997), 35-53.

[Sm5] _ Vanishing theorems, singularities, and effective bounds in algebraic geometry via prime characteristic local algebra, in: Algebraic geometry - Santa Cruz, 1995, J. Kollár, R. Lazarsfeld and David R. Morrison (eds.) Proc. Sympos. Pure Math., 62 (1997), 289-325.

[Ve] J.D. Vélez, Openness of the F-rational locus and smooth base change, J. Algebra, 172 (1995), 425-453.

[Wa1] K.-i. Watanabe, Some remarks concerning Demazure's construction of normal graded rings, Nagoya Math. J., 83 (1981), 203-211.

[Wa2] Study of F-purity in dimension two, in: Algebraic Geometry and Commutative Algebra in honor of Masayoshi Nagata, Vol. II, Kinokuniya, Tokyo, (1988), 791-800.

[Wa3] _ F-regular and F-pure normal grade rings, J. Pure Appl. Algebra, 71 (1991), 341-350.

[Wa4] _ F-purity and F-regularity vs. log-canonical singularities, preprint.

Received June 23, 1998 and revised October 23, 1998.

UNIVERSITY OF UTAH

SAlt Lake City, UT 84112-0090

E-mail address: singh@math.utah.edu 